\title{
EXPLORE MULTIVARIABLE SPATIO-TEMPORAL DATA WITH THE TIME WAVE CASE STUDY ON METEOROLOGICAL DATA
}

\author{
Xia Li ${ }^{\mathrm{a}, \mathrm{b}, *}$, Menno-Jan Kraak ${ }^{\mathrm{a}}$ \\ ${ }^{a}$ ITC- International Institute for GeoInformation Science and Earth Observation \\ PO Box 6, 7500 AA Enschede, the Netherlands-@itc.nl \\ ${ }^{\mathrm{b}}$ College of Earth Science And Resources, Chang’an University
}

Commission II

KEY WORDS: Time wave, Temporal exploration, Time space

\begin{abstract}
:
Traditionally the GIScience community is well able to deal with the locational and attribute component of spatio-temporal data. However, the methods and techniques to deal with the data's temporal component are less developed. This paper introduces a conceptual framework that combines user tasks, available temporal data and visualization theories to discuss temporal visualization. Two limitations of existing method are improved by introducing the time wave environment which is a close combination of temporal graphic representation and temporal interactive tools, and operates in so-called time space. A case study based on meteorological data illustrates the approach.
\end{abstract}

\section{INTRODUCTION}

Many of the most important challenges our society is facing today, such as global climate change, economic development and infectious diseases depend on spatio-temporal data to detect and analyze changes as well as trends to support problem solving. Especially the temporal component of the data should be studied carefully to understand the changes and their impacts. Current data collection techniques offer a wide variety of thematic data in many different spatial and temporal resolutions. From a temporal perspective, earth observation techniques provide data with temporal resolutions varying from weeks, days, hours, to even minutes. The challenge faced is how to process, manage, and use these continuous streams of data to support problem solving and decision making. The application of graphic representations in a dynamic and interactive geovisualization environment is part of the solution.

Geovisualization integrates approaches from disciplines including cartography with those from scientific visualization, image analysis, information visualization, exploratory data analysis and GIScience (Dykes, MacEachren et al. 2005). The graphic representations, mostly maps, are used to stimulate (visual) thinking about geospatial patterns, relationships and trends. This is strengthened by looking at the data in a number of alternative ways. Playing with the data using these multiple representations without constraints (traditions) will trigger the mind of the users, and lead to an increase in their knowledge. This does improve our understanding of how to use visualization to get a better insight into spatial data, but not necessarily in temporal data. This is partly due to the fact that most methods and techniques used to solve the geo-problems are from either location or attribute perspective. This paper will look at the geo-problems from a temporal angle, from what is called time space ( $\mathrm{Li}$ and Kraak 2008). Time space is a visualization space to represent time and answer the temporal questions; for example, what is the temporal distribution of precipitation per month, season or year? How is it linked to location space (e.g. maps) and attributes space (e.g. diagrams).

In the wider context of GIScience research on temporal data analysis and modelling (Goralwalla, Ozsu et al. 1998) and temporal visualization are receiving increased attention (Mackinlay, Robertson et al. 1991; Harrison 1994; Allen 1995; Brown 1998; Harris, Hetzler et al. 2000). This includes papers that offer an overview over existing visualization methods (Andrienko, Andrienko et al. 2003; Aigner, Miksch et al. 2007). Most of these focus on applications in the information visualization field or on discussions of specific aspects of temporal visualization such as static and dynamic representations (Muller 2003), linear time (Silva and Catarci 2000) and the visualization process (Chi 1998). Others take a more overall approach. Aigner et al (Aigner, Miksch et al.) discussed temporal visualization based on a time-oriented framework. Andrienko (Andrienko, Andrienko et al.) focused on spatio-temporal exploration and considers the time visualization from both data and user tasks. However, compared with location space, and attribute space, time space hasn't been studied very well.

In this paper, a proposed solution is to take a closer look at the integration of several visualization theories accepted in GIScience and information visualization, and see how to extend these with a specific temporal component. This is done by analyzing user tasks to structure the temporal data. Hereby the limitations of existing temporal visualization method will be discussed and a specific graphic representation will be suggested. To structure time space the suggested graphic representation should allow the user to represent the temporal data, to display different views on time (e.g. linear or cyclic), and last but not least should allow for interaction. For this the time wave was introduced ( $\mathrm{Li}$ and Kraak 2008). In the next section, a commonly accepted visual problem solving approach is discussed with specific attention for the nature of the data, the

\footnotetext{
* Corresponding author.
} 
user task and the visualization environment. After this background information a temporal conceptual framework for visualization is presented, followed by a case study that demonstrates the alternative approach.

\section{VISUALIZATION THEORY AND TIME SPACE}

\subsection{Visualization theory}

A common approach to support problem solving with visualization is shown in the scheme in Figure 1. A set of tasks, translated into questions is executed in an appropriate visualization environment with suitable data to solve the problem at hand. Three keywords are of importance: user tasks, data framework and visualization framework. The visualization framework includes the graphic representation and the functional tools to 'play' with the graphics.

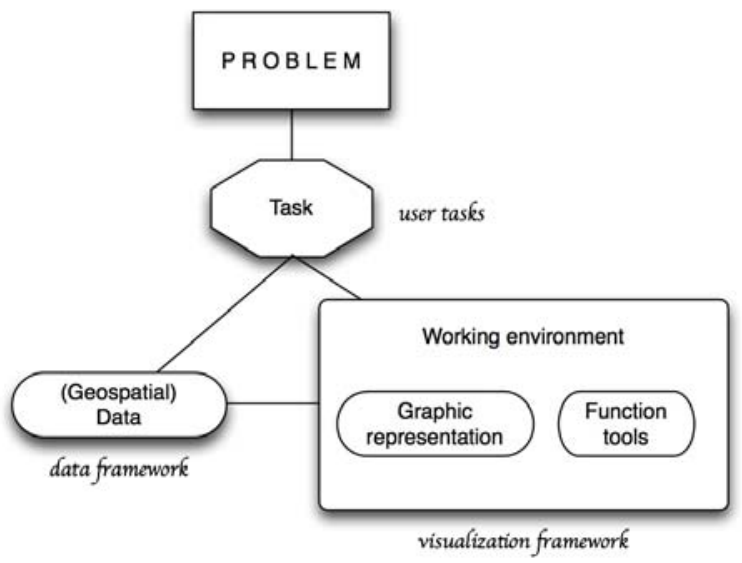

Figure 1. Visual problem solving: The relation between user tasks, a data framework and a visualization framework

Relevant questions are: How to abstract questions based on the data? How to address the data in a visualization environment? How to decide on the right graphic representation(s) and the required functions to answer the questions? Most cartographic and geovisualization theories are studied by looking at the interrelationship of these tightly coupled aspects, such as Bertin (1983), Macrachren (1995), Peuquet (2002) and Andrienko (2006).

\subsection{Time space}

Time space includes temporal graphic representations and temporal interactive tools. Here the requirements of time space will be discussed from temporal data and temporal user tasks perspectives.

\subsubsection{Temporal data and temporal visualization}

Compared with "attribute" and "location", time is considered special. Attribute and location can change over time, but the reverse is not possible. The notion of time is difficult to grasp. This has led to many different views on time. Examples relevant in GISciense are found in Frank(1994), Goralwalla, Ozsu et al. (1998) Peuquet (2002) and Aogner, Miksch et al. (2007) . It can be said time passes continuously, but it is observed and measured in discrete points or intervals. This results in a view of continuous time versus discrete time. Time can also be considered as relative (e.g. last week) or absolute (e.g. May 27th). The continuous nature of time makes it linear (before, after) but it obviously holds cyclic characteristics as well (day, season). The granularity of time is defined by the different units in which time is expressed, and can be used to define the scale of time. These granularities inherit from both linear and cyclic characteristics (hours, seasons).

From the temporal data aspect, the views on time such as linear or cyclic, continuous or discrete, single scale or multiple scale, should be considered and addressed in time space properly. In relation to the task each view might have different requirements for temporal visualization. For example, the question 'how did the city expand?' could be answered with discrete multiple views showing a set of snapshots of the city's extent. Alternatively an animation could be used to answer the same question in continues view. The dynamic nature of the animation could also attract attention to other aspects of the expansion. Based on the character of the data, a linear or cyclic representation might by appropriate. Both could express either continuous or discrete time. Although there might be a straightforward solution in the selection process of the visual representation, it could be useful to try other visualizations as well, because alternative view might reveal patterns or aspects of the data that could remain hidden in the straightforward solution.

\subsubsection{Temporal user tasks and temporal visualization}

Even though the selection of a graphic representation is based upon the nature of the data, the user task plays an important role as well, since at the end, the graphic representation has to answer questions. For instance, the question 'When did countries get their independence?' might require different representations depending on the focus of the question. A timeline with names and years along will do if the temporal distribution is the focus. If one is also interested in the spatial distribution then a world map with labels indicating the year of independence could the most suitable representation. If both are of interest, a space-time-cube (Hägerstrand 1967) could be a possible solution. When one would also be interested in the season of independence the graphic representation should be able to handle both linear and cyclic time and the time wave might be a solution.

Shneiderman's visual information seeking mantra is widely accepted in the visualization field and can be applied in time space. First, the temporal graphic representation is used to locate the time and show the temporal overview, and then temporal interactive tools are required to carry out temporal zooming and temporal filtering options to get the details-ondemand. This process is often an interactive and iterative process, which is supported by a close combination of representation and interactive tools in time space. However, this close combination has not been realized in temporal visualization. There are many temporal graphic representations which could display a temporal pattern of data, such as ThemeRiver (Havre, Hetzler et al. 2000), Stacked bar chat (Harris 1999), People garden (Xiong and Donath 1999), MultiCombs (Tominski, Abello et al. 2003) and etc. To assist answering the questions, temporal interactive tools should allow one to identify single or multiple points in time, identify points at periodic intervals, or define intervals of certain length in both the linear and the cyclic format. Some have experience with this approach, like Koike et al. (1997) with TimeSlider and Edsall et al (1997) experimented with a time wheel query device in the TEMPEST system. Above temporal visualization methods are either a temporal representation or a temporal interactive tool only. 


\section{TEMPORAL VISUALIZATION AND TIME WAVE: AS A NEW APPROACH IN TIME SPACE}

Most temporal representation are either controlled by a timeline (linear time) (Mackinlay, Robertson et al. 1991; Plaisant, Milash et al. 1996; Silva and Catarci 2000; Wijk 2002) or a time wheel (cyclic time) (Mackinlay, Robertson et al. 1994; Carlis and Konstan 1998; Harris 1999; Daassi, Fauvet et al. 2002). However, this does not always result in satisfying solutions because many phenomena have both linear and cyclic characteristics. The time wave (Figure 2; Li and Kraak 2008) is one potential solution for representing both the linear and cyclic nature of data. It is a combination of the timeline and the time wheel and offers an alternative view by its own. With the timeline and the time wheel it is difficult to show multiple time scales. However, the time wave can show multiple time scales by nesting different waves with different wavelengths and amplitudes based on their temporal scale.

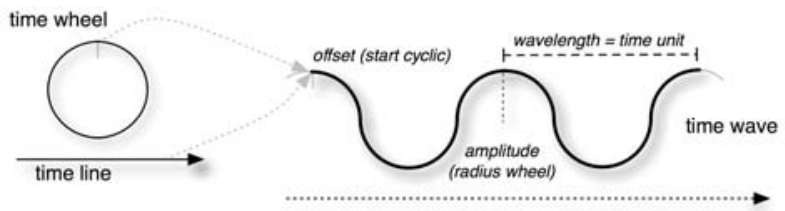

Figure 2. The time wave as a construct of the time line and the time wheel combining the linear and cyclic nature of time $(\mathrm{Li}$ and Kraak 2008)

Time wave not only could show the linear and cyclic time temporal pattern of data as a temporal representation, but also carry out zooming and filter as an interactive tools. For temporal interactive tools, the time wave can do both linear and cyclic operation as well. In addition, the interactive time wave also support one to represent some locational and attribute characteristics in relation with the phenomenon's temporal distribution which can be explored.

Furthermore, the time wave allows users to interact with the temporal reference or temporal representation. Supported by Coordinated Multiple View (CMV) (Roberts 2005; Roberts 2008) principle, one could identify or select any time instant or interval at various scale, or select a temporal pattern, to show the corresponding information in the location space or attribute space. Figure 3 shows an interpretation of time space with the time wave combining the representation and manipulation functions to carry out the interactive and iterative exploration process. The figure also demonstrates the link between time space, location space and attribute space. With a temporal question, one starts in time space and depending on the nature of the question, the answer can be given in time space itself or one 'jumps' out of time space into location or attribute space. For instance, the question related to year of independence of the countries is an example where one moves to location space when one needs to see spatial patterns next to temporal patterns. In answering complex question one might have to jump from space to space in an extensive iterative process to identify and compare temporal, spatial and attributer patterns.

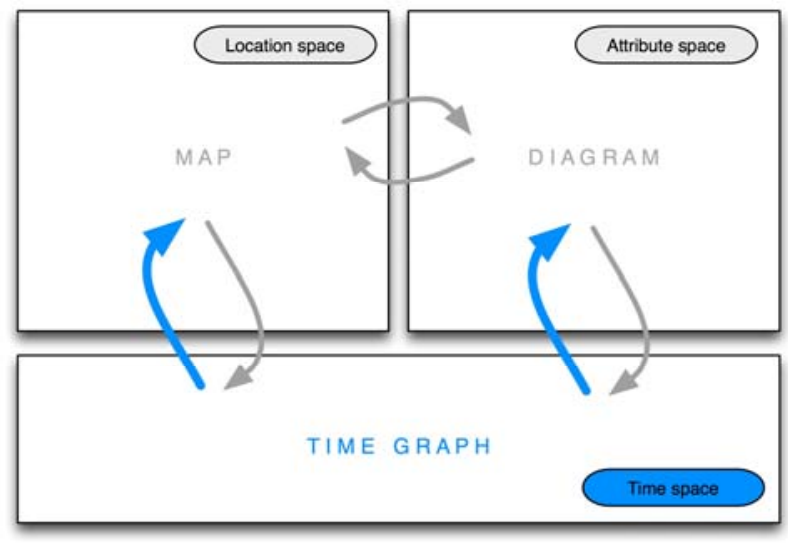

Figure 3. The interactive options to move from time space into location and attribute space supported by CMV principle

\section{CASE STUDY: TIME SPACE AND METEOROLOGICAL DATA}

To see how to create a proper time graph based on the analysis of temporal data, user tasks, a case study is discussed in this section. The data set contains observations of nineteen meteorological stations in Beijing, China. For each station, known at location (x, y, z), the temperature and the dominant land use information are given. The temperature was measured at minute interval over the months in July 2007 and July 2008. A data overview is shown in Figure 4. The location data is point data in context of geographical units (districts in Beijing city); the attribute data is quantitative (temperature) and qualitative (land use); the temporal data has linear characteristics (measurements over a month) and multi-scale cyclic characteristics (July for two year and days and nights for 24 hour periods)

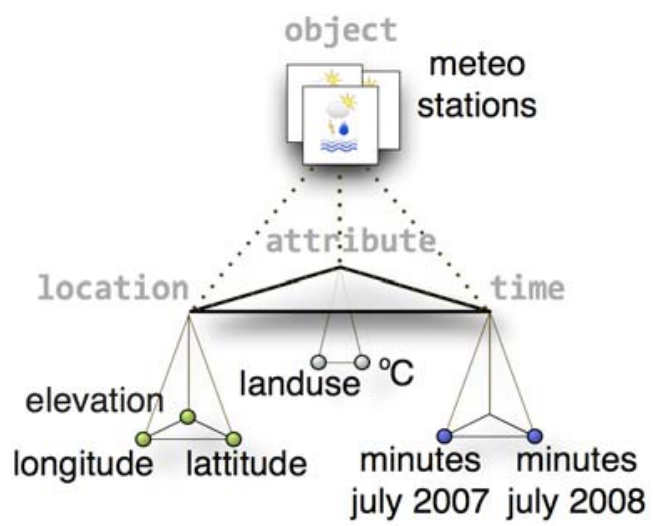

Figure 4. Case study data: the characteristics of the meteorological stations in the Beijing area.

The user task is to find out when the temperature reaches its daily maximum value at the different meteorological stations. Is the pattern related to the longitude, latitude and elevation of each meteorological station? What are the other impact factors, such as land use? Is there any difference between those two years? The temporal questions, such as when, how often, in what order, synchronization and trends, always have a relation to other data components. For instance, from object perspective (station name, station ID), location perspective (height; east/west; north/south), attribute perspective (low, high, or average temperature; land use category like urban, vegetation, water, or other). 
Based on figure 4, figure 5 gives a schematic overview of the coordinated multiple view environment in which time space plays a key role. The time wave is selected as graphic representation since it can handle the temporal data requirements of the case study: linear and cyclic multi scale time. The wave length could be months, weeks, days or any other time unit one would need. It also allows interaction and is directly linked to location and attribute space which is needed if one has to switch spaces while executing the user tasks. In Figure 5 this is represented by the in and out arrows. Location space show the map with the position of the meteorological stations and attribute space shows a scatterplot of the station's temperature versus land use. Figure 6 gives a snapshot of the actual software. The time wave is a plug-in for the Udig open source GIS. The time wave can also include a visual representation of the data's attribute and location characteristics. In Figure 5 symbols related to the meteorological stations, the temperatures observed, as well as land use could be plotted on the wave. Figures 7 and 8 give examples related to the case study.

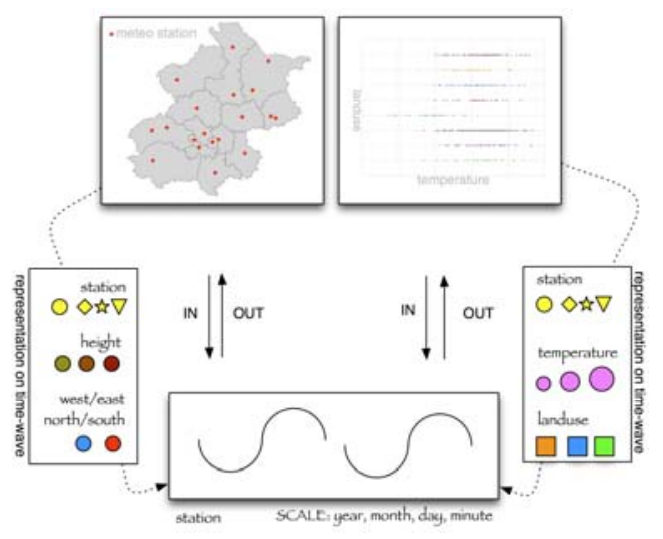

Figure 5. The working environment of the time wave, with options to visually represent characteristics from location and attribute space on the wave

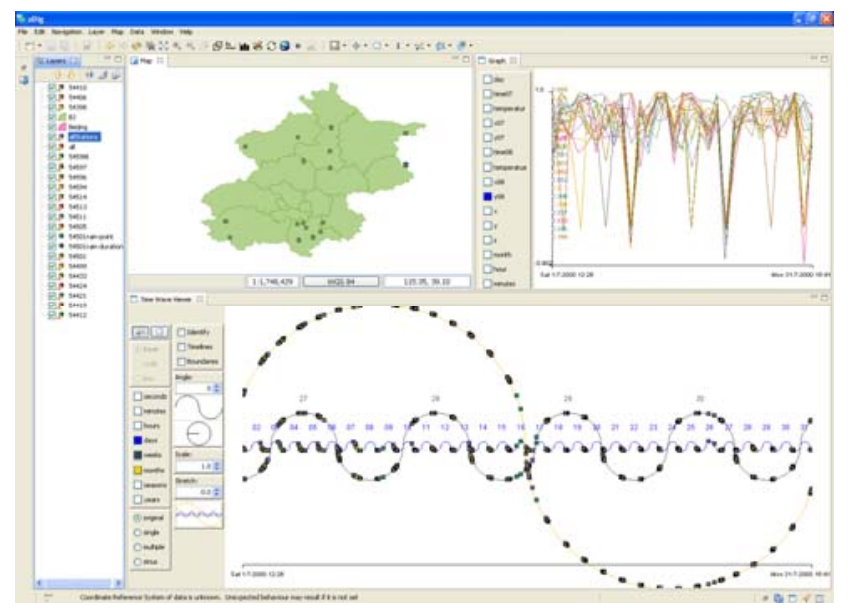

Figure 6. Snapshot of the time space/time wave environment as implemented in the open source GIS uDig

Figure 7 gives two examples of how time space works in practice. In the upper section of the Figure an overview of the month July 2007 is given. The wave length represents a day. For each day and each meteorological station, a symbol is located on the wave at the time when the highest temperature was observed. It is obvious that a wave like this will only offer and overview. A first glance at the data shows that the highest temperature is reached a few hours after 12 noon (the top of the wave). However, both at the beginning and the end of the month an anomaly in the temporal pattern can be observed. These two anomalies have been further explored in the Figure. In both cases one can first zoom in the wave to see more details. At the beginning of the month (the left of the Figure) two stations are rather late in reaching their maximum temperature and at the end of the month (the right of the Figure) there is a single day on which all stations are late in reaching their maximum temperature. In the first situation it makes sense to get more details on those two particular stations, and switch to location space to see if their location is special. The map doesn't reveal anything special, since stations with similar geographic condition can be found. For the second situation it was decided to switch to attribute space and have a closer look at the temperature ranges of all stations at that particular day and the days before and after. It can be observed that the maximum temperature for that particular day was also considerably lower than the days before and after. The available data do not give a good explanation. Other meteorological parameters, for example cloud cover, may be introduced to give an explanation. The experts will have to continue exploring the data and perhaps obtain additional information in order to be able to explain the anomalies.

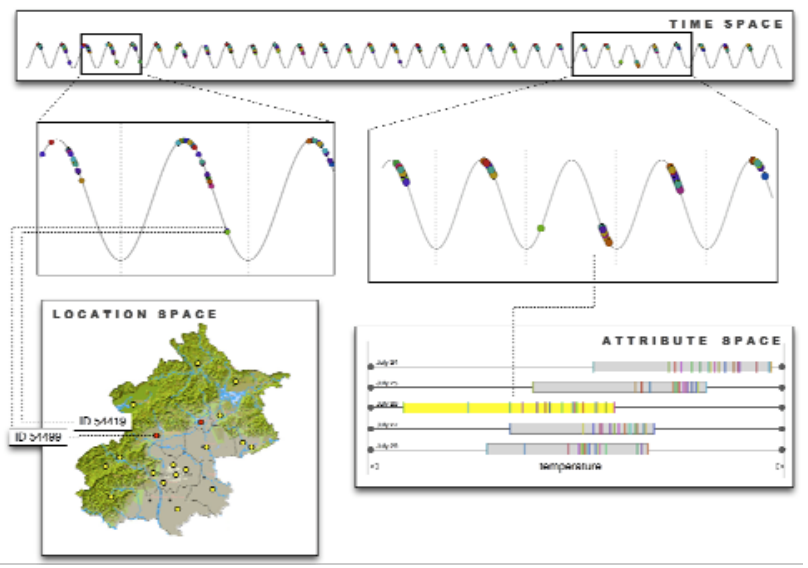

Figure 7. Working with the time wave: identify and explain temporal patterns. At the top the overview of July 2007 showing all stations at the moment these observe the highest temperature. In the middle zooming in on two clear anomalies in the monthly temporal pattern. At the lower left a map in location space and the lower right a temperature diagram in attribute space.

Figure 8 shows another useful function of the time wave. The top wave is the normal time wave, here showing again the moment that the daily temperature reaches its maximum at each meteorological station for three days. It is possible to create a set of parallel waves (Figure 8b). For each meteorological station a separate wave is created to obtain a better view on the patterns. This approach has been derived from the parallel coordinate plot. The wave in Figure 8c shows the data sorted based on the values of July $1^{\text {st }}$. This result in having the station with the earlier highest temperature in the lower wave. Keeping this sort order the following days show a different pattern. It means the order of each stations reach its maximum temperature varies over different day. It is possible to sort the 
data based on any other available variables. For instance, one could sort based on the height of the stations (Figure 8d), or sort from east to west or north to south. After sorting, one might observe patterns that will stimulate one to jump to location or attribute space, to look for more detail, to switch back to overview mode or even to retrieve more data. Working with the time wave to explore temporal patterns is clearly an iterative process. In the next section the specific advantages and possible limits of the approach will be discussed.

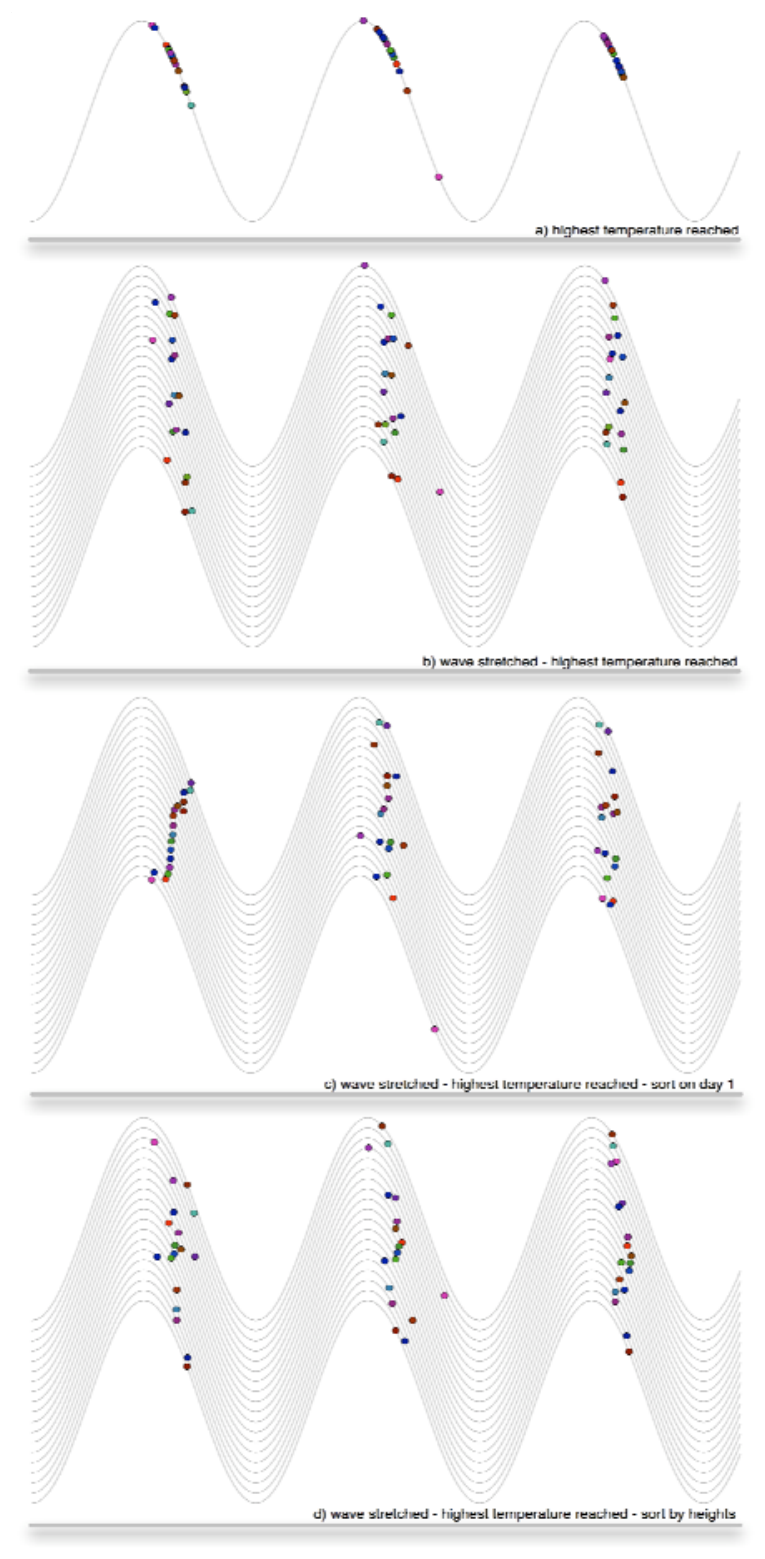

Figure 8. Working with the time wave: comparing temporal patterns. a) an overview of the first three days of July 2008 showing when the maximum temperature was observed for each station; b) temporal stretch by creating a set of parallel waves to un-clutter the pattern as seen in the upper wave; c) sorted wave based on the temperature values of July 1, 2008; d) sorted by station's elevation (highest elevation in lowest parallel wave).

\section{CONCLUSIONS AND DISCUSSIONS}

In this paper an alternative approach to work with spatiotemporal data has been presented based on a visual problem solving approach. The nature of the temporal data and the user task at hand are the driving forces to select a suitable graphic representation. In time space the graphic representation focuses on the temporal aspect of the data. Here the time wave is introduced. It not only combines linear and cyclic time, but also combines temporal data representations and interaction, and allows a limited representation of attribute and location data. The exploratory activities are guided by the visual information seeking mantra of Shneiderman. Depending on the user task, one can switch from one space to the other, and into any of the information seeking modes (overview, zoom / filter, and details on demand).

A case study based on the data observed at meteorological station in Beijing has demonstrated the time space framework and the capabilities of the time wave. Temporal patterns and distribution are studied from time space switching to location space (maps) and attribute space (diagrams) when required. The time wave is a good example of how an alternative view on the data might reveal patterns not always obvious from traditional graphic representations. In overview mode it is a very suitable visualization to provide an impression of the nature in data, being linear or cyclic. In this mode the wave easily reveals anomalies as well, as for instance in figure 7 . This effect can be strengthened by moving the horizontal line ( $\mathrm{x}$-axis) vertically through the wave.

It is not argued that the time wave is the only visual representation in time space. Graphics based on the time line or time wheel only, would be preferred in certain situations. This very much depends on the nature of the data and the task at hand. However, it is claimed that the optimal solution space is time space interactively linked with location and attribute space. This allows the user to flexibly tackle the problem from many different perspectives.

Future work will deal with a detailed validation of the time wave in other case studies and its relation to graphic representation in location and attribute space. Known data sets will be used to see if and how the time wave might discover known patterns and possibly reveal new temporal patterns as well, based on tasks executed by users. In relation to the task space (see figure 3) further work is ongoing in which existing temporal visualization methods are be analyzed on their strength and weakness in the context of the actual temporal problem of users. If successful this could lead to a kind of advisory system that assist the user to select a suitable graphic representation in time space dedicated to the user task one is dealing with.

\section{ACKNOWLEDGEMENTS}

This research Supported by the Programme of Introducing Talents of Discipline to Universities, "111 project" , No: B08039

\section{REFERENCE}

Aigner, W., S. Miksch, et al., 2007. Visualization Timeoriented Data--- A Systematic View. Computers and Graphics, 31, pp.401-409.

Allen, R. B., 1995. Interactive Timelines as Information System Interfaces. Symposium Libraties, Japan. 
Andrienko, G. and N. Andrienko, 2006. Exploratory Analysis of Spatiall and Temporal Data. Springer, Berlin, Germany.

Andrienko, G., N. Andrienko, et al., 2003. Interactive maps for visual exploration of grid and vector geodata. ISPRS Journal of Photogrammetry and Remote Sensing, 57(5-6), pp.380-389.

Andrienko, N., G. Andrienko, et al., 2003. Exploratory spatiotemporal visualization: an analytical review. Journal of Visual Languages \& Computing, 14(6), pp.503-541.

Bertin, J., 1983. Semiology of graphics : diagrams, networks, maps. University of Wisconsin Press, Madison.

Brown, I. M., 1998. A 3D Interface for Visualization of Serial Periodic Data. ACM GIS'98, Washington DC USA.

Carlis, J. V. and J. A. Konstan, 1998. Interactive Visualization of Serial Periodic Data. ACM UIST'98, San Francisco.

Chi, E. H., et al., 1998. Visualizing the Evolution of Web Ecologies. ACM CHI'98, CA USA.

Daassi, C., M.-C. Fauvet, et al., 2002. Multiple Visual Representation of Temporal Data. Proceedings of the 13th International Conference on Database and Expert Systems Applications Springer-Verlag.

Dykes, J., A. M. MacEachren, et al., Eds. 2005. Exploring Geovisualization. Elsevier Science \& Technology Books

Edsall, R., M.-J. Kraak, et al., 1997. Assessing the Effectiveness of Temporal Legends in Environmental Visualization GIS/LIS '97 Annual Conference and Exposition Cincinnati, Ohio

Frank, A., 1994. Different Types of "Times" in GIS. GIS and Computational Science Perspectives.

Goralwalla, I. A., M. T. Ozsu, et al., 1998. An Object-Oriented Framework for Temporal Data Models. Temporal Database-Research and Practice. O. Etzion, S. Jajodia and S. Sripada. Berlin Heidenberg, Springer. 1: pp.1-35.

Hägerstrand, T., 1967. Spatial Process. University of Chicago, Chicago.

Harris, R. L., 1999. Information graphics: a comprehensive illustrated reference Oxford University Press US New York

Harris, S., B. Hetzler, et al., 2000. ThemeRiver: Visualization Theme Changes Over Time. IEEE Symposium on Information Visualization, USA, IEEE Computer Society.

Harrison, B. L., R.Owen, R.M.Baecker, 1994. Timelines: An Interactive System for the Collection of Visualization of Temporal Data. Graphics Interface' 1994, Canadian.

Havre, S., B. Hetzler, et al., 2000. ThemeRiver: Visualizing theme changes over time. IEEE Symposium on Information Visualization, Los Alamitos, USA, IEEE Computer Society.

Koike, Y., A. Sugiura, et al., 1997. TimeSlider: An Interface to Specify Time Point Proceedings of UIST '97.

Li, X. and M.-J. Kraak, 2008. The Time Wave. A New Method of Visual Exploration of Geo-data in Time-space. The Cartographic Journal, 45(3), pp.1-9.

MacEachren, A. M., 1995. How Maps Work: Representation, Visualization, and Design. Guilford press, New York, USA.

Mackinlay, J., G. Robertson, et al., 1994. Developing calendar visualizers for the information visualizer. Proceedings of the 1990 ACM conference on Computer-supported cooperative work, Los Angeles,.
Mackinlay, J. D., G. G. Robertson, et al., 1991. The Perspective Wall: Detail and Context Smoothly Integrated. ACM CHI'91, New York.

Muller, W., Hetdrun Schumann, 2003. Visualization Methods for Time-dependent data-Overview. Winter simulation conference 2003, New Orleans USA.

Peuquet, D. J., 2002. Representations of Space and Time. Guilford, New York.

Plaisant, C., B. Milash, et al., 1996. LifeLines: visualizing personal histories. Conference on Human Factors in Computing Systems Vancouver, Canada ACM

Roberts, J. C., 2005. Exploratory Visualization with Multiple Linked Views. Exploring Geovisualization. J. Dykes, M. A.M. and M.J.Kraak. London, Elsevier. 8: pp.159180.

Roberts, J. C., 2008. Coordinated Multiple Views for Exploratory GeoVisualization. Geographic Visualization: Concepts, Tools and Applications. M. Dodge, M. Mcderby and M. Turner. Chichester, John Wiley \& Sons Inc. 3: pp.25-48.

Silva, S. F. and T. Catarci, 2000. Visualization of Linear Timeoriented data: a survey. Web Information Systems Engineering, 2000.

Tominski, C., J. Abello, et al., 2003. Interactive Poster: AxesBased Visualizations for Time Series Data. Poster Compendium of IEEE Symposium on Information Visualization, IEEE.

Wijk, J. v., 2002. Image based flow visualization. SIGGRAPH, San Antonio, USA.

Xiong, R. and J. Donath, 1999. PeopleGarden: Creating Data Portraits for Users. 12th Annual ACM Symposium on User Interface Software and Technology (UIST '99), NC; USA. 